\title{
Shared Genetic Factors Influence Amygdala Volumes and Risk for Alcoholism
}

\begin{abstract}
Alecia D Dager*, ${ }^{1,2}$, D Reese McKay ${ }^{1,2}$, Jack W Kent Jr ${ }^{3}$, Joanne E Curran ${ }^{3}$, Emma Knowles ${ }^{1,2}$, Emma Sprooten 1,2, Harald HH Göring ${ }^{3}$, Thomas D Dyer ${ }^{3}$, Godfrey D Pearlson ${ }^{1,2}$, Rene L Olvera ${ }^{4}$, Peter T Fox ${ }^{5,6}$, William R Lovallo ${ }^{7}$, Ravi Duggirala ${ }^{3}$, Laura Almasy ${ }^{3}$, John Blangero ${ }^{3}$ and David C Glahn ${ }^{1,2}$

'Olin Neuropsychiatry Research Center, Institute of Living, Hartford Hospital, Hartford, CT, USA; ' Department of Psychiatry, Yale University School of Medicine, New Haven, CT, USA; ${ }^{2}$ Department of Genetics, Texas Biomedical Research Institute, San Antonio, TX, USA; ${ }^{4}$ Department of Psychiatry, University of Texas Health Science Center San Antonio, San Antonio, TX, USA; ${ }^{5}$ Research Imaging Institute, University of Texas Health Science Center San Antonio, San Antonio, TX, USA; ' ${ }^{6}$ South Texas Veterans Health System, San Antonio, TX, USA; 'Department of Psychiatry, Oklahoma University Heath Science Center and Veterans Administration Medical Center, Oklahoma City, OK, USA
\end{abstract}

Alcohol abuse and dependence (alcohol use disorders, AUDs) are associated with brain shrinkage. Subcortical structures including the amygdala, hippocampus, ventral striatum, dorsal striatum, and thalamus subserve reward functioning and may be particularly vulnerable to alcohol-related damage. These structures may also show pre-existing deficits impacting the development and maintenance of AUD. It remains unclear whether there are common genetic features underlying both subcortical volumes and AUD. In this study, structural brain images were acquired from 872 Mexican-American individuals from extended pedigrees. Subcortical volumes were obtained using FreeSurfer, and quantitative genetic analyses were performed in SOLAR. We hypothesized the following: ( I) reduced subcortical volumes in individuals with lifetime AUD relative to unrelated controls; (2) reduced subcortical volumes in individuals with current relative to past AUD; (3) in non-AUD individuals, reduced subcortical volumes in those with a family history of AUD compared to those without; and (4) evidence for common genetic underpinnings (pleiotropy) between AUD risk and subcortical volumes. Results showed that individuals with lifetime AUD showed larger ventricular and smaller amygdala volumes compared to non-AUD individuals. For the amygdala, there were no differences in volume between current vs past AUD, and non-AUD individuals with a family history of AUD demonstrated reductions compared to those with no such family history. Finally, amygdala volume was genetically correlated with the risk for AUD. Together, these results suggest that reduced amygdala volume reflects a pre-existing difference rather than alcohol-induced neurotoxic damage. Our genetic correlation analysis provides evidence for a common genetic factor underlying both reduced amygdala volumes and AUD risk.

Neuropsychopharmacology (20I5) 40, 4I2-420; do: I 0.1038/npp.20 I4. I87; published online 3 September 20I4

\section{INTRODUCTION}

Chronic alcohol abuse and dependence (alcohol use disorders, AUDs) have been consistently associated with substantial structural brain changes. Recent automated neuroanatomic parcellation approaches have improved the efficiency of analyses in large samples and generally replicated earlier studies, suggesting particular susceptibility of frontal lobes and limbic regions to excessive alcohol exposure (for a review, see Buhler and Mann, 2011). There is specific interest in better characterizing subcortical structures that subserve reward functioning, including the amygdala, hippocampus, dorsal and ventral striatum, and thalamus, as these regions may be linked to initiation and

*Correspondence: Dr AD Dager, Olin Neuropsychiatry Research Center, Institute of Living, Hartford Hospital, 200 Retreat Avenue, Whitehall Building, Hartford, CT 06106, USA, Tel: +I 860545 7670, Fax: + I 860545 7797, E-mail: alecia.dager@yale.edu

Received 20 January 20 I4; revised and accepted 3 June 2014; accepted article preview online 31 July 2014 maintenance of heavy drinking, as well as with consequences of heavy drinking. In particular, addiction models posit that as addiction progresses these circuits become hyperreactive to drug reinforcers, driving motivation, and compulsive drug seeking (Volkow et al, 2011). Several lines of evidence suggest that subcortical structures may be especially vulnerable to alcohol-related damage (Buhler and Mann, 2011; Jernigan et al, 1991), possibly contributing to maintaining heavy drinking. Yet it is less clear whether there may also be genetically linked pre-existing structural differences in these regions, heightening the risk for initiation and maintenance of AUD. In the current study, we used neuroimaging and genetics analyses to characterize the genetic and alcohol-related influences on subcortical structural abnormalities.

Adults with AUD show reduced subcortical volumes (Buhler and Mann, 2011; Jernigan et al, 1991), and several studies attempt to determine whether these abnormalities normalize with extended abstinence. Evidence of subcortical differences between those who maintain abstinence and those who relapse could indicate either that these 
structures are amenable to recovery or that they are involved in enabling abstinence. Cardenas et al (2007) examined changes in brain structure among alcohol-dependent individuals 8 months after the treatment. Those who maintained abstinence showed volumetric recovery of several regions, including thalamus, compared to those who returned to heavy drinking. Although the small sample size of eight individuals who relapsed may limit the ability to detect effects, drinking severity was not related to structural changes. The authors concluded that the observed structural decrements were not influenced by alcohol exposure, rather that these regions enabled abstinence.

Others contend that subcortical regions show little normalization with abstinence, indicating either that these structures are permanently damaged by alcohol exposure or that these differences were pre-existing and potentially reflect genetic predisposition to alcoholism. For instance, a crosssectional voxel-based morphometry investigation showed smaller amygdala volumes among alcoholics who had been abstinent for an average of 7 years, and no relationship between amygdala volume and abstinence duration (Fein et al, 2006), pointing either to pre-existing abnormalities or to lack of normalization with extended sobriety. Later work confirmed this finding, reporting that reduced left amygdala volumes in alcoholism were unrelated to duration of sobriety, and that this reduction was primarily restricted to the basolateral amygdala, a region that has a central role in incentive salience (Makris et al, 2008), possibly reflecting pre-existing reward pathway decrements. Durazzo et al (2011) reported that individuals who relapsed $(n=51)$ and abstained $(n=24)$ had similar baseline deficits in amygdala and hippocampal volumes compared with controls, and smaller baseline amygdala and hippocampal volumes were associated with greater post-treatment alcohol consumption among those who relapsed. A prospective study examined subcortical volumetric changes 6 months following treatment, reporting that reduced amygdala volume predicted craving and subsequent relapse, but not duration of abstinence (Wrase et al, 2008). In contrast, hippocampal and ventral-striatal volumes did not recover with abstinence and may reflect long-term neurotoxicity that resists recovery (Wrase et al, 2008). In a similar study, those who relapsed and those who abstained showed comparable reductions in amygdala and ventral-striatal volume compared with controls (Beck et al, 2012). Together, these studies demonstrate that AUD-related reduced amygdala volume does not recover with abstinence and is associated with subsequent drinking, indicating that amygdala deviations may be preexisting and potentially contributing to AUD risk. Alternatively, hippocampal reductions may reflect neurotoxic damage that does not recover with abstinence.

Other work demonstrates structural irregularities among youths at risk for AUD who have not yet initiated heavy drinking, thereby depicting pre-existing effects that are not confounded by alcohol exposure. AUD shows substantial genetic influence, and individuals with a family history of alcoholism (FHP) are at increased risk for developing AUD (Schuckit, 1985). Attempts to characterize genetically influenced pre-existing volumetric differences in those at risk for AUD typically focus on FHP nondrinking youths. Nondrinking FHP adolescent males from multiplex alcoholism families have smaller right amygdala volumes compared with family history-negative (FHN) youths, but similar left amygdala and hippocampal volumes (Hill et al, 2001), a finding replicated in older adolescents/emerging adults (Hill et al, 2013). Others observe similar hippocampal volumes between FHP and FHN youths (Hanson et al, 2010). Another report identified reduced amygdala, hippocampus, and thalamus volumes in FHP youths (Benegal et al, 2007). In general, these findings corroborate the conclusions drawn from adult literature, suggesting that hippocampal aberrations observed in AUD reflect alcoholrelated neurotoxicity, but that amygdala abnormalities may be pre-existing.

There is mounting evidence that smaller amygdala volumes observed in alcoholism are not associated with the neurotoxic effects of heavy drinking but appear to be sensitive to the genetic risk for illness. In contrast, reductions in other subcortical structures may reflect alcohol-related toxicity. Thus, it is possible that the same genetic factors that influence amygdala volume may also influence AUD risk (eg, pleiotropy). To test this hypothesis, large-scale imaging studies that include individuals with current and past AUD diagnoses and their non-AUD family members are required. Specifically, if a genetic correlation between AUD risk and the volume of a subcortical region can be established, then this suggests that the volume of that brain region is influenced by the same genetic factors that predispose AUD. Here, we examined subcortical volumes, in relationship to AUD diagnosis, in 872 Mexican-American individuals from a large extended pedigree who participated in the Genetics of Brain Structure and Function study. Probands were randomly ascertained from census tracts in San Antonio and all of their available family members were recruited. When comparing subcortical volumes, we hypothesized the following:

1. Reduced volumes of subcortical brain structures in individuals with a lifetime AUD diagnosis relative to unrelated controls, suggesting structural differences associated with AUD.

2. Reduced volumes of subcortical brain structures in individuals with current AUD relative to those with past AUD, suggesting either that subcortical regions recover with abstinence or that larger subcortical regions subserve abstinence.

3. Reduced volumes of subcortical brain structures in nonAUD individuals with a family history of alcoholism compared with non-AUD individuals with no such family history, suggesting genetically related abnormalities.

4. Evidence for genetic correlation indicating that common genetic factors influence both AUD and reduced subcortical volumes (pleiotropy between AUD and subcortical volumes).

\section{MATERIALS AND METHODS}

\section{Participants}

Eight hundred and seventy-two Mexican-American individuals from extended pedigrees participated in the study. Participants were $64 \%$ female, ranging in age from 18 to 85 years old (43.66 years, SD 14.9). Individuals were randomly 
selected from the community with the criteria that they are of Mexican-American ancestry, part of a large family, and live in the area of San Antonio, TX (see the Supplement for recruitment details). Exclusion criteria for the study were history of neurological disorders and MRI contraindications. All participants provided written informed consent, approved by the Institutional Review Boards at the University of Texas Health Science Center San Antonio (UTHSCSA) and Yale University.

\section{Diagnostic Assessment}

AUD diagnoses were ascertained with the Mini-International Neuropsychiatric Interview (Sheehan et al, 1998), a semi-structured clinical interview that characterizes current (past 12 months) and lifetime DSM-IV symptomatology for alcohol abuse and dependence. Masters- and doctorate-level research staff conducted interviews and participants with possible diagnoses were discussed in case conferences with licensed psychologists or psychiatrists.

\section{Image Acquisition and Processing}

Structural MRI scans were acquired on a 3-Tesla Siemens Trio scanner with an 8-channel head coil in the Research Imaging Institute, University of Texas Health Science Center San Antonio (Siemens, Erlangen, Germany). T1weighted images of $800 \mu \mathrm{m}$ isotropic resolution were acquired using a retrospective motion-correction protocol (Kochunov et al, 2006). The protocol comprised seven T1weighted, three-dimensional TurboFLASH acquisitions with the following parameters: echo time $=3.04 \mathrm{~ms}$, repetition time $=2100 \mathrm{~ms}$, inversion time $=785 \mathrm{~ms}$, flip angle $=113^{\circ}$. Each subject's T1-weighted images were averaged, registered to a Talairach compliant template (Kochunov et al, 2002), and resliced to isotropic $800 \mu \mathrm{m}$ spacing using a three-dimensional, 15-voxel-wide sinc interpolation kernel (Jenkinson et al, 2002).

Subcortical image analyses were conducted with FreeSurfer (http://surfer.nmr.mgh.harvard.edu/) (Dale et al, 1999; Fischl et al, 1999), as implemented in our group (Winkler et al, 2010). As can be seen in Figure 1, each subject's average T1-weighted image was segmented into gray matter volumes for seven subcortical regions (averaged across both hemispheres): hippocampus, amygdala, thalamus, globus pallidus, caudate, putamen, and ventral diencephalon (Fischl et al, 2002). Although there is debate in the field regarding manual $v s$ automatic anatomic segmentation, FreeSurfer measurements have been validated against histological analysis (Rosas et al, 2002) and manual measurements (Kuperberg et al, 2003; Salat et al, 2004), and show reliability across scanners and field strengths (Han et al, 2006).

\section{Quantitative Genetic Analyses}

Maximum likelihood variance decomposition methods, as implemented in SOLAR (http://www.txbiomed.org/departments/genetics/genetics-detail?r=37) (Almasy and Blangero, 1998), were used for all genetic analyses. Briefly, the covariance matrix $\Omega$ for a pedigree of individuals is given by $\Omega=2 \Phi \sigma^{2}{ }_{\mathrm{G}}+I \sigma_{\mathrm{E}}^{2}$, where $\sigma_{\mathrm{G}}^{2}$ is the genetic variance due to the additive genetic factors, $\Phi$ is the kinship matrix representing the pair-wise kinship coefficients among all individuals, $\sigma_{\mathrm{E}}^{2}$ is the variance due to environmental effects, and $I$ is an identity matrix. Kinship coefficients were confirmed with molecular genetic testing, producing a multigenerational pedigree that summarizes the genetic relationships among all individuals. See previous work by Blangero et al (2001) for more details.

Heritability $\left(h^{2}\right)$ is the proportion of total phenotypic variance $\left(\sigma_{p}^{2}\right)$ accounted for additive genetic factors by contrasting the observed phenotypic covariance matrix with the covariance matrix predicted by kinship $\left(h^{2}=\sigma^{2}{ }_{\mathrm{G}} / \sigma^{2}{ }_{\mathrm{p}}\right)$. High heritability indicates that a large part of the phenotypic variance is explained by the genetic proximity between individuals, such that the more closely related individuals are, the more similar they are for that trait. The significance of heritability estimates was tested by comparing the $\log _{e}$ likelihood of the model in which $\sigma^{2}{ }_{G}$ is constrained to zero with that of a model in which $\sigma^{2}{ }_{G}$ is estimated. Twice the difference between the two $\log _{e}$ likelihoods of these models yields a test statistic that is asymptotically distributed as a $1 / 2: 1 / 2$ mixture of a $\chi^{2}$ variable and a point mass at zero. We used a polygenic model that estimated the influence of specific variables (additive genetic variation, covariates and random unidentified environmental effects) calculating heritability and its significance ( $p$-value).

Bivariate genetic correlation analyses were performed to test if common genetic factors influnce two traits. Bivariate analyses decompose phenotypic correlations $\left(\rho_{\mathrm{P}}\right)$ between two traits into genetic $\left(\rho_{\mathrm{G}}\right)$ and environmental $\left(\rho_{\mathrm{E}}\right)$ correlations, accounting for kinship: $\rho_{\mathrm{P}}=\rho_{\mathrm{G}} \sqrt{ }\left(h^{2}{ }_{\mathrm{A}} h^{2} \mathrm{~B}\right)+$ $\rho_{\mathrm{E}} \sqrt{ }\left[\left(1-h^{2}{ }_{\mathrm{A}}\right)\left(1-h_{\mathrm{B}}^{2}\right)\right]$, where $h^{2}{ }_{\mathrm{A}}$ and $h_{\mathrm{B}}^{2}$ are the heritability estimates for traits $\mathrm{A}$ and $\mathrm{B}$. The magnitude of $\rho_{\mathrm{G}}$ is a measurement of pleiotropy or shared genetic variance between traits (Almasy et al, 1997). Pleiotropy is the degree to which the same genes contribute to both trait $\mathrm{A}$ and $\mathrm{B}$ (ie, both AUD and subcortical volumes). The significance of genetic and environmental correlation was tested by comparing the $\log _{\mathrm{e}}$ likelihood for two restricted models (with either $\rho_{\mathrm{G}}$ or $\rho_{\mathrm{E}}$ constrained to zero) against the log likelihood for the model in which these parameters were estimated. A significant genetic correlation is evidence for pleiotropy, suggesting that a gene or set of genes jointly influences both phenotypes (Almasy et al, 1997).

Demographic variables including age, age ${ }^{2}$ and sex were used as covariates in all analyses. In addition, to control for global differences in head size, intracranial volume was similarly used as a covariate. To correct for multiple comparisons, a false discovery rate of $5 \%$ was applied.

\section{RESULTS}

\section{Sample}

Two-hundred and ninety-seven individuals met the criteria for a lifetime AUD diagnosis (34\% of the sample, see Table 1). Of these, 188 met the criteria for current alcohol dependence or abuse and 109 were remitted. The remaining sample had no past or current AUD diagnosis and were grouped as follows: unaffected 1st degree relatives $(n=137$, $16 \%$ of the sample); unaffected 2 nd through 5 th degree 

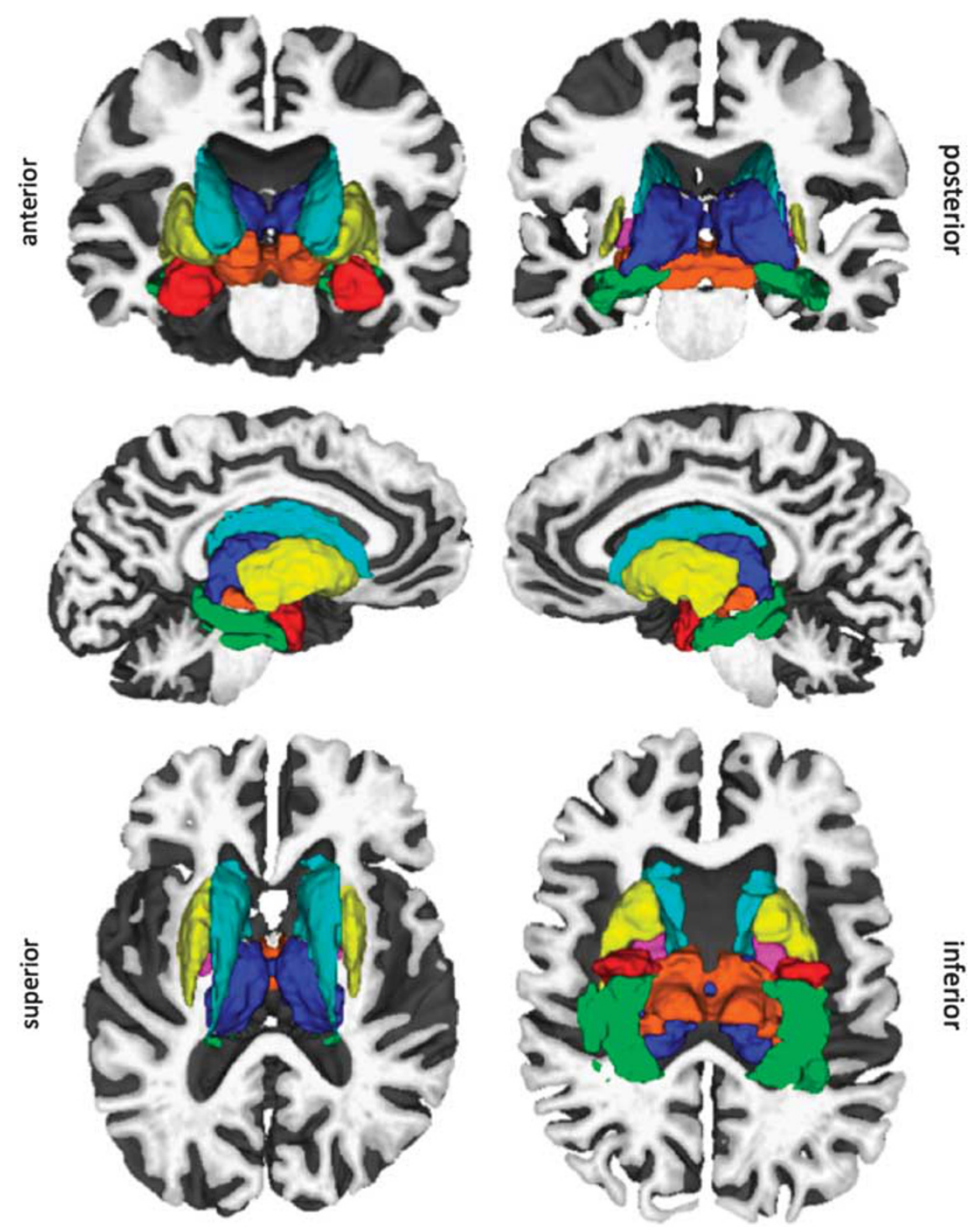

Figure I Segmentation of subcortical nuclei in a candidate subject: amygdala (red), hippocampus (green), thalamus (blue), pallidum (magenta), caudate (cyan), putamen (yellow), ventral diencephalon (orange). Mango software (http://rii.uthscsa.edu/mango//mango.html) was used to visualize structures.

relatives $(n=211,24 \%$ of the sample); or unrelated individuals without family history of AUD ( $n=227,26 \%$ of the sample). The members of this final group are referred to as 'unaffected, unrelated controls' in subsequent analyses as they have no personal or family history of AUD.

As can be seen in Table 1, individuals with a lifetime AUD were far more likely to be male $\left(\chi^{2}=116.53, \mathrm{df}=1, p=3.6\right.$ $\left.\times 10^{-27}\right)$. Indeed, individuals with a lifetime history of AUD were far more likely to be male than unaffected, unrelated controls $\left(\chi^{2}=40.79, \mathrm{df}=1, p=1.7 \times 10^{-10}\right)$, their unaffected 1 st degree relatives $\left(\chi^{2}=75.34, \mathrm{df}=1, p=4.0\right.$ $\left.\times 10^{-18}\right)$ or their unaffected $2-5$ th degree relatives $\left(\chi^{2}=69.87, \mathrm{df}=1, p=6.3 \times 10^{-17}\right)$. In contrast, there were more females with a current AUD compared to individuals with a past $\operatorname{AUD}\left(\chi^{2}=6.05, \mathrm{df}=1, p=0.01\right)$. Although the mean age did not differ between individuals with a lifetime AUD and the remaining sample as a whole $\left(\chi^{2}=1.55, \mathrm{df}=1\right.$, $p=0.21$ ), individuals with AUD were significantly younger than their unaffected 1 st degree relatives $\left(\chi^{2}=53.83, \mathrm{df}=1\right.$, $\left.p=1.7 \times 10^{-13}\right)$.

As age, age ${ }^{2}$, sex, and intracranial volume were associated with total gray matter volume, $\left(\chi^{2}=582.19, \mathrm{df}=1, p=1.3\right.$ $\times 10^{-128}, \chi^{2}=3.73, \mathrm{df}=1, \quad p=0.05, \chi^{2}=31.36, \mathrm{df}=1$, $p=2.1 \times 10^{-8}$ and $\chi^{2}=599.10, \mathrm{df}=1, p=2.62 \times 10^{-132}$, respectively) these variables were included as covariates in subsequent analyses.

\section{Heritability}

All subcortical brain regions demonstrated significant heritability (Table 2). AUD was significantly heritable in this sample $\left(h^{2}=0.282\right.$, standard error $\left.=0.123, p=0.006\right)$, while controlling for age $(p=0.74), \operatorname{age}^{2}(p=0.0001)$, sex $\left(p=3.25 \times 10^{-15}\right)$, and intracranial volume $(p=0.051)$.

Hypothesis 1: Lifetime AUD vs unaffected, unrelated controls. After controlling for age, sex and intracranial 
Table I Sample Characteristics $(n=872)$

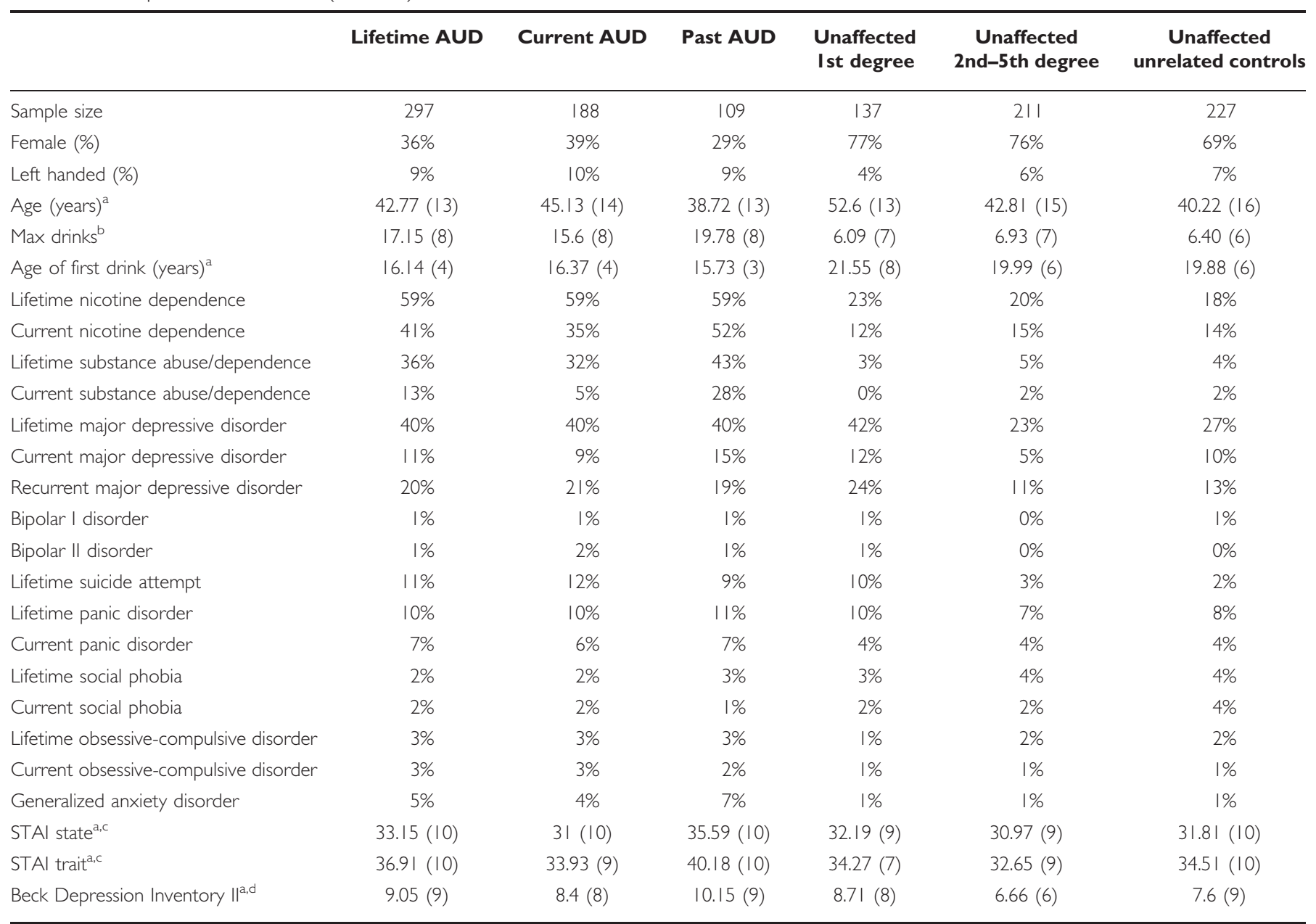

${ }^{\mathrm{a}}$ Mean (SD).

bMaximum lifetime drinks in $24 \mathrm{~h}$, Mean (SD).

'State-Trait Anxiety Inventory (STAl) (Spielberger et al, 1970).

${ }^{\mathrm{d}}$ Beck et al (1996).

Table 2 Heritability Estimates for Subcortical Brain Regions

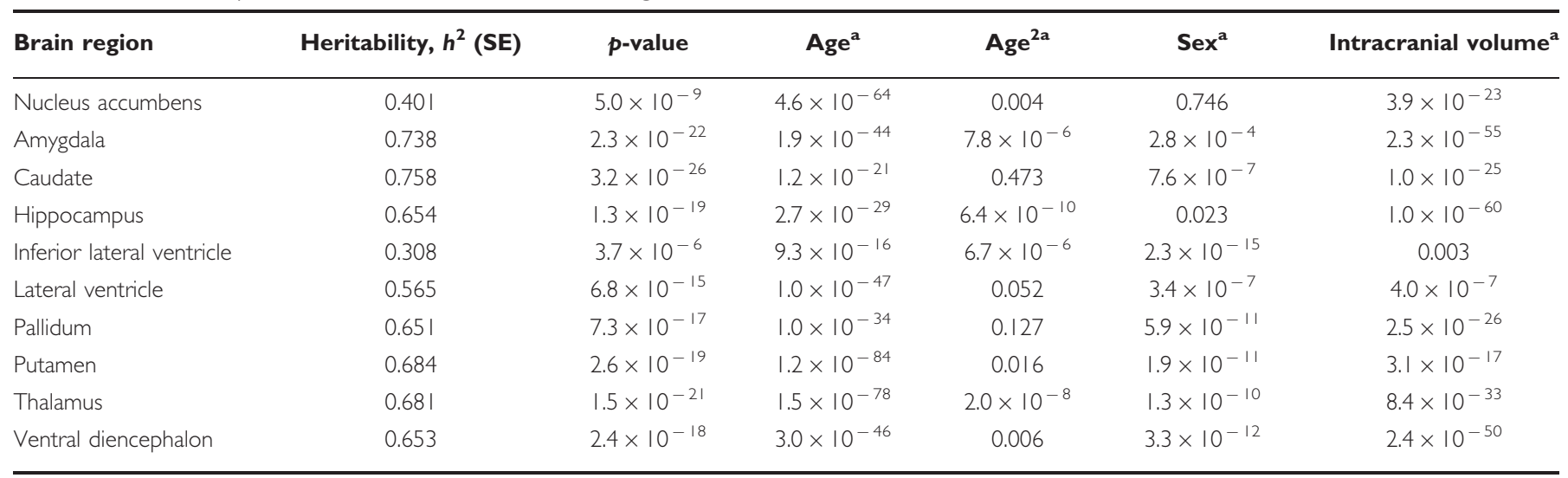

${ }^{a} p$-value for the specific covariate analysis (eg, beta $p$-values). All heritabilities were significant after correction for multiple comparisons.

volume, individuals with a lifetime AUD had larger inferior lateral ventricles when compared with unaffected, unrelated controls (Cohen's $d=0.26$; see Table 3 and Figure 2).
Similarly, individuals with a lifetime AUD had larger inferior lateral ventricles when compared with their unaffected 1 st degree $(d=0.24)$ and more distantly related 
Table 3 Association between Subcortical Volumes and Lifetime AUD

\begin{tabular}{|c|c|c|c|c|}
\hline Brain region & $\begin{array}{l}\text { Lifetime AUD vs unaffected, } \\
\text { unrelated controls }{ }^{\mathrm{a}}\end{array}$ & $\begin{array}{l}\text { Lifetime AUD vs unaffected } \\
\text { Ist degree relative }{ }^{\mathrm{a}}\end{array}$ & $\begin{array}{l}\text { Lifetime AUD vs Unaffected } \\
2-5 \text { th degree relative }\end{array}$ & $\begin{array}{l}\text { Past AUD vs } \\
\text { Current AUD }\end{array}$ \\
\hline Nucleus accumbens & $0.202, p=0.653$ & $0.453, p=0.501$ & $1.290, p=0.256$ & $0.154, p=0.695$ \\
\hline Caudate & $0.221, p=0.638$ & $0.438, p=0.508$ & $0.165, p=0.685$ & $0.933, p=0.334$ \\
\hline Hippocampus & $0.089, p=0.765$ & $0.003, p=0.956$ & $3.457, p=0.063$ & $0.736, p=0.391$ \\
\hline Pallidum & $0.014, p=0.907$ & $2.690, p=0.101$ & $4.135, p=0.042$ & $1.284, p=0.257$ \\
\hline Putamen & $0.581, p=0.446$ & $0.531, p=0.466$ & $2.484, p=0.115$ & $1.965, p=0.161$ \\
\hline Thalamus & $0.182, p=0.670$ & $2.304, p=0.129$ & $6.038, p=0.014$ & $2.443, p=0.118$ \\
\hline Ventral diencephalon & $1.133, p=0.287$ & $0.243, p=0.622$ & $3.085, p=0.079$ & $2.189, p=0.139$ \\
\hline
\end{tabular}

${ }^{a} \chi^{2}, p$-value, bolded cells were significant after correction for multiple comparisons.

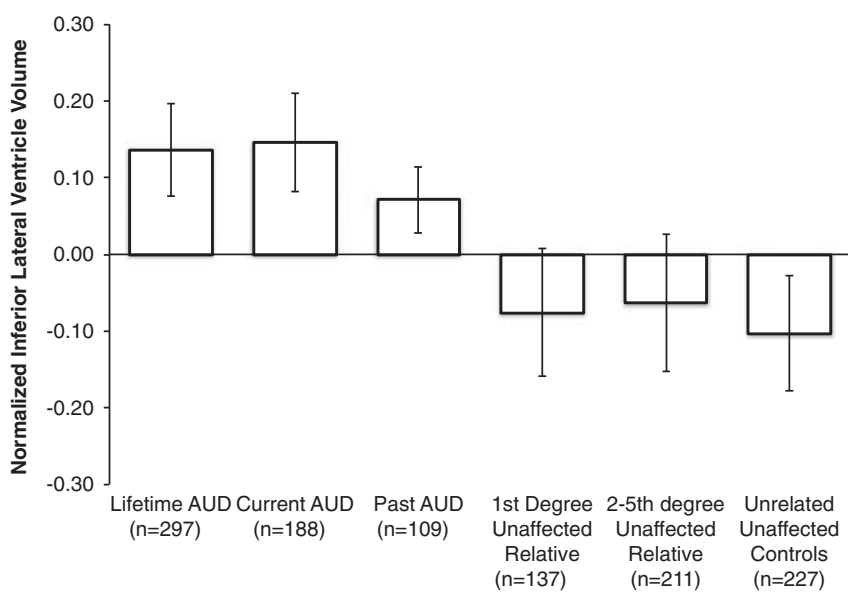

Figure 2 Average inferior lateral ventricle volumes (inverse Gaussian normalized) between groups, after controlling for age, age ${ }^{2}$, sex and intracranial volume.

relatives $(d=0.16)$. Individuals with a lifetime AUD had smaller amygdala volumes when compared with unaffected, unrelated controls after controlling for demographic variables and head size $(d=0.39$; see Figure 3$)$.

Hypothesis 2: Current vs past AUD. There was no difference in amygdala volume between individuals with current $v s$ past AUD $\left(\chi^{2}=2.08, p=0.149\right)$. Both groups showed similar differences in amygdala volumes compared with controls (current $\chi^{2}=3.46, p=0.063, d=0.15$; past $\chi^{2}=6.47, p=0.001, d=0.34$ ). In contrast, individuals with current AUD had larger inferior-ventricular volumes than those with past $\operatorname{AUD}\left(\chi^{2}=6.47, p=0.001, d=0.28\right)$.

Hypothesis 3: Unaffected family history positive vs unaffected, unrelated controls. Unaffected 1st degree relatives of individuals with an AUD had smaller amygdala volumes compared with unaffected, unrelated controls $\left(\chi^{2}=4.45, p=0.035, d=0.22\right)$. No other subcortical brain region differed between unaffected 1st degree relatives and unaffected, unrelated controls ( $p$-values ranged between 0.140-0.954).

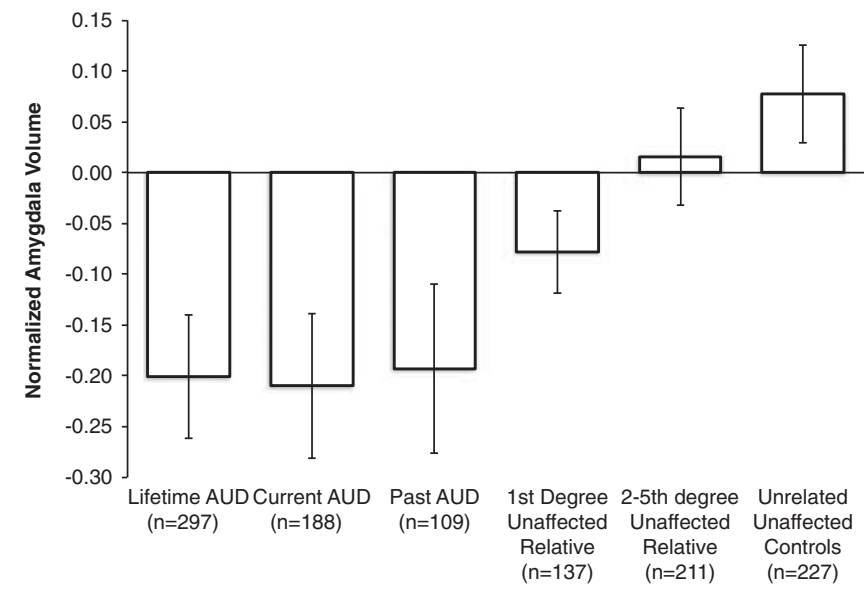

Figure 3 Average amygdala volumes (inverse Gaussian normalized) between groups, after controlling for age, age ${ }^{2}$, sex and intracranial volume.

Hypothesis 4: Pleiotropy: alcoholism risk and amygdala volume. The genetic correlation between amygdala volume and AUD was $\rho_{\mathrm{g}}=-0.267, p=0.045$, suggests common genetic factors are involved in amygdala volume and risk for AUD. The endophenotype ranking value (Glahn et al, 2012) for AUD and amygdala was $E R V=0.113$. In contrast, the environmental correlation was non-significant $\left(\rho_{\mathrm{e}}=\right.$ $0.087, p=0.42$ ), indicating that there was no shared environmental effect associated with both AUD and amygdala volume.

\section{Co-morbidities}

Rates of co-morbid nicotine dependence, drug use disorders, and mood disorders are shown in Table 1, and discussed further in the Supplement. Briefly, these traits were heritable but not related to amygdala volume, whereas substance use disorders and maximum lifetime drinks were associated with ventricle volume (see Supplementary Results). 


\section{DISCUSSION}

The current study examined the shared genetic and environmental underpinnings of AUD and subcortical structural volumes. We found that (1) individuals with lifetime diagnoses of AUD demonstrated larger ventricle volumes and smaller amygdala volumes compared with unrelated controls; (2) individuals with past AUD demonstrated similar reductions in amygdala volumes compared to those with current AUD; (3) among those with no personal history of AUD, FHP individuals showed smaller amygdala volumes than FHN individuals; and (4) there is evidence that shared genetic factors influence AUD and amygdala volume.

Our heritability estimates were similar to those found in previous studies, highlighting the robustness of our phenotypic procedures. We estimated the heritability of AUD as $h^{2}=0.282$, which corresponds to our previous estimate of 0.30 in an overlapping cohort (Olvera et al, 2011). In contrast, others have reported somewhat higher heritability of 0.56 for alcohol dependence in twin samples of predominantly European ancestry (Goldman et al, 2005). This variation in heritability between studies could reflect differences in diagnostic classification, ethnic differences in genetic influence over AUD risk, or cultural influences on alcohol use patterns in the Hispanic community (Arroyo et al, 2003; Caetano and Clark, 1998). We found relatively high heritability estimates for subcortical volumes, ranging from 0.39 for the nucleus accumbens to 0.76 for the caudate. These results are consistent with our previous reports (McKay et al, 2013; Winkler et al, 2010) and a recent metaanalysis that confirmed high heritability for subcortical volumes, despite wide variability in subcortical heritability estimates between studies (Blokland et al, 2012).

Individuals with AUD demonstrated larger ventricles and smaller amygdala volumes compared with unaffected, unrelated controls, corroborating existing evidence of volumetric decrements associated with alcoholism (Beck et al, 2012; Buhler and Mann, 2011; Jernigan et al, 1991; Makris et al, 2008). Consistent with previous studies, we found that individuals with current AUD had larger ventricles than those with past AUD, suggesting that the enlarged ventricles in alcoholism may recover with abstinence (Pfefferbaum et al, 1998). FHP individuals did not show ventricular enlargement, nor did we find evidence of pleiotropy for ventricular volume and AUD $\left(\rho_{\mathrm{g}}=-0.077, p=0.657\right)$, indicating that ventricular enlargement could be a direct or indirect consequence of current heavy alcohol exposure. The maximum number of drinks in a 24 -h period was also associated with ventricular enlargement, lending further support to the argument that ventricle size is compromised by alcohol-related toxicity.

We observed similar amygdala reductions for both current and past AUD diagnoses. Others have demonstrated that amygdala decrements are not related to the duration of abstinence (Durazzo et al, 2011; Fein et al, 2006; Makris et al, 2008; Wrase et al, 2008). One interpretation of this finding is that amygdala volumes are permanently damaged by alcohol-related neurotoxicity, and do not normalize with extended sobriety. Alternatively, individuals with current and past AUD may show similar amygdala reductions because these abnormalities are pre-existing. In particular, among individuals with no personal history of AUD, we demonstrated smaller amygdala volumes in FHP compared with FHN individuals. This result directly parallels work describing amygdala decrements in FHP nondrinking youths (Benegal et al, 2007; Hill et al, 2001) and emerging adults (Hill et al, 2013), providing evidence of genetically related pre-existing amygdala abnormalities, which could partially underlie vulnerability for AUD, placing individuals at risk for initiation and escalation of drinking (Hill et al, 2001). However, previous human studies did not formally identify an underlying genetic link between the amygdala and risk for AUD. In contrast, our work clearly demonstrates pleiotropy, suggesting shared genetic underpinnings of amygdala volume and AUD. Moreover, we found no common environmental factor contributing to amygdala volume and AUD. Together, these findings support the hypothesis that amygdala abnormalities are pre-existing and not reduced by alcohol exposure or other overlapping environmental risks (Hanson et al, 2010; Hill et al, 2001).

Structural abnormalities in the amygdala may contribute to addiction risk (Koob, 1999), yet the mechanism of this risk is unclear as there is little work directly linking amygdala structure to the subsequent development of heavy drinking. In rats, central amygdala lesions diminished voluntary ethanol consumption (Moller et al, 1997), highlighting the importance of intact amygdala and associated connections in relation to drinking. The amygdala may have a direct role in alcohol-specific learning and motivation, but may also subserve behaviors that are more general risks for addictive behaviors, including emotion regulation and externalizing behaviors.

Extensive work has implicated amygdala function in the development and maintenance of addiction, particularly through its role in drug-related learning and motivation (Volkow et al, 2011; Zahr and Sullivan, 2008). The amygdala is thought to subserve affective response to drug-related stimuli and promote the acquisition of drug-associated positive and negative reinforcement (Koob, 1999). For instance, fMRI studies of individuals with AUD suggest that amygdala response to alcohol cues reflects emotional salience and motivation for alcohol-related stimuli (Dager et al, 2013; Schneider et al, 2001; Tapert et al, 2003), which could drive the development and maintenance of heavy use. In alcohol-dependent adults, smaller amygdala volumes predicted greater craving and subsequent relapse following treatment (Wrase et al, 2008) and reduced amygdala volumes in FHP nondrinking youths may underlie faster acquisition of alcohol-related cue-response learning (Hill et al, 2001). Regarding this, we previously demonstrated enhanced amygdala fMRI response to repeated presentation of alcohol cues among FHP individuals, which could indicate a genetically linked sensitivity of the amygdala to alcohol cues, contributing to alcoholism risk (Dager et al, 2013). There is also recent evidence that amygdala response to alcohol cues is genetically mediated (Jorde et al, 2013).

In addition to its proposed role in alcohol cue-response learning and alcohol craving, the amygdala may also confer alcoholism risk through its involvement in affective regulation (Tessner and Hill, 2010). In support of this, a recent longitudinal study reported that smaller amygdala volumes were associated with higher self-reported negative affectivity at baseline and more subsequent alcohol-related 
problems (Cheetham et al, 2014). Smaller amygdala volumes may contribute to alcoholism risk via increased externalizing symptoms, such as impulsivity (Benegal et al, 2007; Tessner and Hill, 2010). FHP non-AUD participants, aged 8-24 years, demonstrated smaller amygdala volumes compared to FHN youths, and greater amygdala deficits were associated with more externalizing symptomatology (Benegal et al, 2007).

When corrected for intracranial volume, we found no evidence for reductions of other subcortical structures in individuals with current AUD, contrasting work identifying thalamic and hippocampal abnormalities in AUD (Buhler and Mann, 2011; Jernigan et al, 1991). In the current study, participants were recruited from the community rather than a treatment setting, and included individuals with alcohol abuse or dependence. Thus, our participants may have included those with less severe alcohol problems than previous studies, leading to fewer structural abnormalities (Buhler and Mann, 2011). In addition, previous studies often included only men, or may not have controlled for sex and age in the same way as the current study; age and sex are important factors in understanding the influence of chronic alcoholism on brain structure (Buhler and Mann, 2011). Structural analysis methods have also differed between studies and may impact results.

One advantage of our extended pedigree design is that it enables us to disentangle genetic variance from environmental factors. However, this sample may also limit our ability to generalize to wider populations. Our AUD participants could differ from those seeking treatment, particularly with respect to motivation. We did not examine the influence of demographic variables such as socioeconomic status or education level, which are important factors to investigate in future studies. We did not ascertain detailed information on maternal substance use during pregnancy. No participant demonstrated fetal alcohol syndrome, yet we are not able to rule out the possible effects of prenatal substance exposure in this sample. Importantly, our amygdala results were not related to nicotine dependence, other substance use disorders, or mood disorders (see Supplementary Materials).

In sum, we demonstrate reduced amygdala volumes among individuals with current or past AUD, regardless of abstinence duration, as well as FHP individuals with no personal history of AUD. Together, these results suggest that reduced amygdala volume reflects a pre-existing difference rather than alcohol-induced neurotoxic damage. Moreover, our genetic correlation analyses provides evidence for a common genetic factor contributing to reduced amygdala volumes and to alcoholism, further indicating the existence of genetically mediated pre-existing amygdala abnormalities that contribute to AUD.

\section{FUNDING AND DISCLOSURE}

The authors declare no conflict of interest.

\section{ACKNOWLEDGEMENTS}

We thank the participants of the San Antonio Family Study and our research staff. Financial support for this study was provided by the National Institute of Mental Health (grant numbers: MH0708143 (Principal Investigator (PI): DCG), MH078111 (PI: JB), and MH083824 (PI: DCG and JB)). Theoretical development of SOLAR is supported by National Institute of Mental Health (grant number: MH59490) (PI: JB). The supercomputing facilities used for this work at the AT\&T Genomics Computing Center were supported, in part, by a gift from the AT\&T Foundation and by the National Center for Research Resources (grant number: S10 RR029392).

\section{REFERENCES}

Almasy L, Blangero J (1998). Multipoint quantitative-trait linkage analysis in general pedigrees. Am J Hum Genet 62: 1198-1211.

Almasy L, Dyer TD, Blangero J (1997). Bivariate quantitative trait linkage analysis: pleiotropy versus co-incident linkages. Genet Epidemiol 14: 953-958.

Arroyo JA, Miller WR, Tonigan JS (2003). The influence of Hispanic ethnicity on long-term outcome in three alcoholtreatment modalities. J Stud Alcohol 64: 98-104.

Beck A, Wustenberg T, Genauck A, Wrase J, Schlagenhauf F, Smolka MN et al (2012). Effect of brain structure, brain function, and brain connectivity on relapse in alcohol-dependent patients. Arch Gen Psychiatry 69: 842-852.

Beck AT, Steer RA, Brown GK (1996). Manual for the Beck Depression Inventory-II. Psychological Corp: San Antonio, TX.

Benegal V, Antony G, Venkatasubramanian G, Jayakumar PN (2007). Gray matter volume abnormalities and externalizing symptoms in subjects at high risk for alcohol dependence. Addict Biol 12: 122-132.

Blangero J, Williams JT, Almasy L (2001). Variance component methods for detecting complex trait loci. Adv Genet 42: 151-181.

Blokland GA, de Zubicaray GI, McMahon KL, Wright MJ (2012). Genetic and environmental influences on neuroimaging phenotypes: a meta-analytical perspective on twin imaging studies. Twin Res Hum Genet 15: 351-371.

Buhler M, Mann K (2011). Alcohol and the human brain: a systematic review of different neuroimaging methods. Alcohol Clin Exp Res 35: 1771-1793.

Caetano R, Clark CL (1998). Trends in alcohol-related problems among whites, blacks, and Hispanics: 1984-1995. Alcohol Clin Exp Res 22: 534-538.

Cardenas VA, Studholme C, Gazdzinski S, Durazzo TC, Meyerhoff DJ (2007). Deformation-based morphometry of brain changes in alcohol dependence and abstinence. Neuroimage 34: 879-887.

Cheetham A, Allen NB, Whittle S, Simmons J, Yucel M, Lubman DI (2014). Volumetric differences in the anterior cingulate cortex prospectively predict alcohol-related problems in adolescence. Psychopharmacology (Berl) 231: 1731-1742.

Dager AD, Anderson BM, Stevens MC, Pulido C, Rosen R, Jiantonio-Kelly RE et al (2013). Influence of alcohol use and family history of alcoholism on neural response to alcohol cues in college drinkers. Alcohol Clin Exp Res 37(Suppl 1): E161-E171.

Dale AM, Fischl B, Sereno MI (1999). Cortical surface-based analysis. I. Segmentation and surface reconstruction. Neuroimage 9: 179-194.

Durazzo TC, Tosun D, Buckley S, Gazdzinski S, Mon A, Fryer SL et al (2011). Cortical thickness, surface area, and volume of the brain reward system in alcohol dependence: relationships to relapse and extended abstinence. Alcohol Clin Exp Res 35: 1187-1200.

Fein G, Landman B, Tran H, McGillivray S, Finn P, Barakos J et al (2006). Brain atrophy in long-term abstinent alcoholics who 
demonstrate impairment on a simulated gambling task. Neuroimage 32: 1465-1471.

Fischl B, Salat DH, Busa E, Albert M, Dieterich M, Haselgrove C et al (2002). Whole brain segmentation: automated labeling of neuroanatomical structures in the human brain. Neuron 33: 341-355.

Fischl B, Sereno MI, Dale AM (1999). Cortical surface-based analysis. II: inflation, flattening, and a surface-based coordinate system. Neuroimage 9: 195-207.

Glahn DC, Curran JE, Winkler AM, Carless MA, Kent JW Jr, Charlesworth JC et al (2012). High dimensional endophenotype ranking in the search for major depression risk genes. Biol Psychiatry 71: 6-14.

Goldman D, Oroszi G, Ducci F (2005). The genetics of addictions: uncovering the genes. Nat Rev Genet 6: 521-532.

Han X, Jovicich J, Salat D, van der Kouwe A, Quinn B, Czanner S et al (2006). Reliability of MRI-derived measurements of human cerebral cortical thickness: the effects of field strength, scanner upgrade and manufacturer. Neuroimage 32: 180-194.

Hanson KL, Medina KL, Nagel BJ, Spadoni AD, Gorlick A, Tapert SF (2010). Hippocampal volumes in adolescents with and without a family history of alcoholism. Am J Drug Alcohol Abuse 36: 161-167.

Hill SY, De Bellis MD, Keshavan MS, Lowers L, Shen S, Hall J et al (2001). Right amygdala volume in adolescent and young adult offspring from families at high risk for developing alcoholism. Biol Psychiatry 49: 894-905.

Hill SY, Wang S, Carter H, McDermott MD, Zezza N, Stiffler S (2013). Amygdala volume in offspring from multiplex for alcohol dependence families: the moderating influence of childhood environment and 5-HTTLPR Variation. J Alcohol Drug Depend S1.

Jenkinson M, Bannister P, Brady M, Smith S (2002). Improved optimization for the robust and accurate linear registration and motion correction of brain images. Neuroimage 17: 825-841.

Jernigan TL, Schafer K, Butters N, Cermak LS (1991). Magnetic resonance imaging of alcoholic Korsakoff patients. Neuropsychopharmacology 4: 175-186.

Jorde A, Bach P, Witt SH, Becker K, Reinhard I, Vollstadt-Klein S et al (2013). Genetic variation in the atrial natriuretic peptide transcription factor GATA4 modulates amygdala responsiveness in alcohol dependence. Biol Psychiatry 75: 790-797.

Kochunov P, Lancaster J, Thompson P, Toga AW, Brewer P, Hardies $J$ et al (2002). An optimized individual target brain in the Talairach coordinate system. Neuroimage 17: 922-927.

Kochunov P, Lancaster JL, Glahn DC, Purdy D, Laird AR, Gao F et al (2006). Retrospective motion correction protocol for highresolution anatomical MRI. Hum Brain Mapp 27: 957-962.

Koob GF (1999). The role of the striatopallidal and extended amygdala systems in drug addiction. Ann N Y Acad Sci 877: 445-460.

Kuperberg GR, Broome MR, McGuire PK, David AS, Eddy M, Ozawa F et al (2003). Regionally localized thinning of the cerebral cortex in schizophrenia. Arch Gen Psychiatry 60: $878-888$.
Makris N, Oscar-Berman M, Jaffin SK, Hodge SM, Kennedy DN, Caviness VS et al (2008). Decreased volume of the brain reward system in alcoholism. Biol Psychiatry 64: 192-202.

McKay DR, Knowles EE, Winkler AA, Sprooten E, Kochunov P, Olvera RL et al (2013). Influence of age, sex and genetic factors on the human brain. Brain Imaging Behav 8: 143-152.

Moller C, Wiklund L, Sommer W, Thorsell A, Heilig M (1997). Decreased experimental anxiety and voluntary ethanol consumption in rats following central but not basolateral amygdala lesions. Brain Res 760: 94-101.

Olvera RL, Bearden CE, Velligan DI, Almasy L, Carless MA, Curran JE et al (2011). Common genetic influences on depression, alcohol, and substance use disorders in Mexican-American families. Am J Med Genet B Neuropsychiatr Genet 156B: 561-568.

Pfefferbaum A, Sullivan EV, Rosenbloom MJ, Mathalon DH, Lim KO (1998). A controlled study of cortical gray matter and ventricular changes in alcoholic men over a 5-year interval. Arch Gen Psychiatry 55: 905-912.

Rosas HD, Liu AK, Hersch S, Glessner M, Ferrante RJ, Salat DH et al (2002). Regional and progressive thinning of the cortical ribbon in Huntington's disease. Neurology 58: 695-701.

Salat DH, Buckner RL, Snyder AZ, Greve DN, Desikan RS, Busa E et al (2004). Thinning of the cerebral cortex in aging. Cereb Cortex 14: 721-730.

Schneider F, Habel U, Wagner M, Franke P, Salloum JB, Shah NJ et al (2001). Subcortical correlates of craving in recently abstinent alcoholic patients. Am J Psychiatry 158: 1075-1083.

Schuckit MA (1985). Studies of populations at high risk for alcoholism. Psychiatr Dev 3: 31-63.

Sheehan DV, Lecrubier Y, Sheehan KH, Amorim P, Janavs J, Weiller E et al (1998). The Mini-International Neuropsychiatric Interview (M.I.N.I.): the development and validation of a structured diagnostic psychiatric interview for DSM-IV and ICD-10. J Clin Psychiatry 59(Suppl 20): 22-33 quiz 34-57.

Spielberger CD, Gorsuch RL, Lushene RE (1970). Manual for the State-Trait Anxiety Inventory. Consulting Psychologists Press: Palo Alto, CA.

Tapert SF, Cheung EH, Brown GG, Frank LR, Paulus MP, Schweinsburg AD et al (2003). Neural response to alcohol stimuli in adolescents with alcohol use disorder. Arc Gen Psychiatry 60: 727-735.

Tessner KD, Hill SY (2010). Neural circuitry associated with risk for alcohol use disorders. Neuropsychol Rev 20: 1-20.

Volkow ND, Wang GJ, Fowler JS, Tomasi D, Telang F (2011). Addiction: beyond dopamine reward circuitry. Proc Natl Acad Sci USA 108: 15037-15042.

Winkler AM, Kochunov P, Blangero J, Almasy L, Zilles K, Fox PT et al (2010). Cortical thickness or grey matter volume? The importance of selecting the phenotype for imaging genetics studies. Neuroimage 53: 1135-1146.

Wrase J, Makris N, Braus DF, Mann K, Smolka MN, Kennedy DN et al (2008). Amygdala volume associated with alcohol abuse relapse and craving. Am J Psychiatry 165: 1179-1184.

Zahr NM, Sullivan EV (2008). Translational studies of alcoholism: bridging the gap. Alcohol Res Health 31: 215-230.

Supplementary Information accompanies the paper on the Neuropsychopharmacology website (http://www.nature.com/npp) 